\title{
Aspects of Flat/CCFT Correspondence
}

\author{
Reza Fareghbal ${ }^{a, b}$, Ali Naseh ${ }^{b}$ \\ ${ }^{a}$ Department of Physics, Shahid Beheshti University, G.C., Evin, Tehran 19839, Iran. \\ ${ }^{b}$ School of Particles and Accelerators, Institute for Research in Fundamental Sciences \\ (IPM), P.O. Box 19395-5531, Tehran, Iran \\ E-mails: r_fareghbal@sbu.ac.ir, naseh@ipm.ir
}

\begin{abstract}
Flat/CCFT is a correspondence between gravity in asymptotically flat backgrounds and a field theory which is given by contraction of conformal field theory. In order to find a dictionary for Flat/CCFT correspondence one can start from the AdS/CFT and take the contraction of CFT in the boundary as the dual description of the flat-space limit (zero cosmological constant limit) of the asymptotically AdS spacetimes in the bulk side. In this paper we show that the Cardy-like formula of $\mathrm{CCFT}_{2}$ is given by contraction of a proper formula in the $\mathrm{CFT}_{2}$. This formula is the modified Cardy formula which gives the entropy of inner horizon of BTZ black holes.
\end{abstract}




\section{Introduction}

A possible way for generalizing the gauge/gravity duality for asymptotically flat spacetimes is using the AdS/CFT correspondence and taking flat-space limit (zero cosmological constant limit) of asymptotically AdS spacetimes and trying to find a proper interpretation of this limit in the boundary CFT. A proposal for this method has been introduced in [1, 2] where the flat-space limit of the bulk corresponds to the contraction of boundary CFT. The contracted conformal field theory (CCFT) which is dual to asymptotically flat spacetimes has the same symmetry as the asymptotic symmetry of the bulk geometry.

The correspondence between the flat-space limit in the bulk and contraction of the dual boundary theory can be used in defining a dictionary for the Flat/CCFT correspondence. The first steps in this direction have already done in papers [1, 2] which showed that the Bondi-Metzner-Sachs (BMS) algebra [3, 4] as the asymptotic symmetry of asymptotically flat spacetimes at null infinity can be obtained by contraction of the Virasoro algebras as the symmetry of the two-dimensional CFT. Another important work in this context is [5] where a Cardy-like formula for the $\mathrm{CCFT}_{2}$ which is dual to the three-dimensional asymptotically flat spacetimes, has been introduced. The Cardy-like formula as an estimation of the CCFT degeneracy of states, gives the exact entropy of the cosmological horizon of the three dimensional cosmological solutions. These geometries are the shifted-boost orbifold of the three-dimensional Minkowski spacetimes and can be found by taking the flat-spce limit from the BTZ black holes. [6] .

Defining a field theory just by contracting a CFT has some advantages. Its correlation functions are given by contraction of correlation functions of the parent CFT [7] . This fact can be also used for finding quasi local stress tensor of the asymptotically flat spacetimes. This idea has been followed in paper [8] for the three dimensional asymptotically flat spacetimes and a stress tensor was introduced which gives correct charges for the gravity solutions. The stress tensor of [8] can be also used for computing the $\mathrm{BMS}_{3}$ charge algebra.

The approach of using a contracted CFT in flat-space holography has been explored in many works. For example it can be used for finding higher-spin theories in three-dimensional asymptotically flat spacetimes [9, 10]. The reader can find a complete list of references in recent work [11] where the current status of problem and also future directions have been mentioned.

The point which we address in this paper, is the relation between the $\mathrm{CCFT}_{2}$ Cardy-like formula and the modified Cardy formula of $\mathrm{CFT}_{2}$. The Cardy-like formula of CCFT has been derived in [5] by defining partition function of CCFT and demanding its invariance 
under some novel modular transformations. In this paper we show that it is simply given by contracting a proper formula of the CFT. This formula is not the known Cardy formula which according to $\mathrm{AdS}_{3} / \mathrm{CFT}_{2}$ correspondence gives the correct entropy of the outer horizon of the BTZ black holes but it is the formula which gives the entropy of the inner horizon [12, 13]. We elaborate on this point in the main text by using the flat-space limit of the BTZ black holes in the bulk side.

The organization of this paper is as follows: In the next section we briefly review the Flat/CCFT correspondence. The main point which we want to clarify in this section is the correspondence between the flat-space limit in the bulk and contraction of the CFT in the boundary. In Section 3 we find the Cardy-like formula of $\mathrm{CCFT}_{2}$ by contracting its counterpart in the parent CFT. Last section is devoted to conclusions and possible future directions.

\section{A Brief Review of the Flat/CCFT Correspondence}

Let us consider three-dimensional Einstein gravity with negative cosmological constant,

$$
S=\frac{1}{16 \pi G} \int d^{3} x \sqrt{-g}\left(R+\frac{2}{\ell^{2}}\right) .
$$

The flat-space limit for this theory is defined by taking zero cosmological constant limit which is given by $\ell \rightarrow \infty$ limit. In order to make this limit well-defined we define the flatspace limit by using the dimensionless parameter $G / \ell$ and sending it to zero while keeping $G$ fixed. At the level of metric, the flat-space limit is gauge dependent. An appropriate gauge that can capture properly the flat-space limit of the asymptotically locally AdS spacetimes is known as BMS gauge [14 16]. For the asymptotically locally $A d S_{3}$ spacetimes the general solution of equations of motions in the BMS gauge can be written as [16]

$$
d s^{2}=\left(-\frac{r^{2}}{l^{2}}+\mathcal{M}\right) d u^{2}-2 d u d r+2 \mathcal{N} d u d \phi+r^{2} d \phi^{2}
$$

where $u$ is the retarded time coordinate, and $\mathcal{M}$ and $\mathcal{N}$ are functions of $u, \phi$ coordinates. Using the equations of motion, one then finds

$$
\partial_{u} \mathcal{M}=\frac{2}{l^{2}} \partial_{\phi} \mathcal{N}, \quad 2 \partial_{u} \mathcal{N}=\partial_{\phi} \mathcal{M}
$$

It is shown in [8] that by proper expansion of functions $\mathcal{M}$ and $\mathcal{N}$ with respect to $G / l$, 
one can find the general asymptotically flat metric as 1

$$
d s^{2}=M d u^{2}-2 d u d r+2 N d u d \phi+r^{2} d \phi^{2}
$$

where

$$
M=\lim _{G / \ell \rightarrow 0} \mathcal{M}=\theta(\phi), \quad N=\lim _{G / \ell \rightarrow 0} \mathcal{N}=\chi(\phi)+\frac{u}{2} \theta^{\prime}(\phi) .
$$

The gauge (2.2) implies an asymptotic symmetry algebra which is given by two copies of the Virasoro algebra [16]:

$$
\left[\mathcal{L}_{m}, \mathcal{L}_{n}\right]=(m-n) \mathcal{L}_{m+n}, \quad\left[\overline{\mathcal{L}}_{m}, \overline{\mathcal{L}}_{n}\right]=(m-n) \overline{\mathcal{L}}_{m+n}, \quad\left[\mathcal{L}_{m}, \overline{\mathcal{L}}_{n}\right]=0
$$

In [16], the authors found the central extension of the surface charges algebra, computed with respect to $\mathrm{AdS}_{3}$ background, with $c=\bar{c}=3 l / 2 G$.

Using (2.6) and explicit form of the generators $\mathcal{L}_{n}$ and $\overline{\mathcal{L}}_{n}$ introduced in [16], one can easily check that the new generators

$$
L_{n}=\mathcal{L}_{n}-\overline{\mathcal{L}}_{-n}, \quad M_{n}=\frac{G}{l}\left(\mathcal{L}_{n}+\overline{\mathcal{L}}_{-n}\right),
$$

in the $G / l \rightarrow 0$ limit results in the $\mathrm{BMS}_{3}$ algebra

$$
\left[L_{m}, L_{n}\right]=(m-n) L_{m+n}, \quad\left[L_{m}, M_{n}\right]=(m-n) M_{m+n}, \quad\left[M_{m}, M_{n}\right]=0,
$$

which is the asymptotic symmetry of the three-dimensional asymptotically flat spacetimes [4]. Moreover, the $\mathrm{BMS}_{3}$ charge algebra contains two central charges which are given by [4]

$$
C_{L L}=\lim _{\frac{G}{l} \rightarrow 0} \frac{c-\bar{c}}{12}, \quad C_{L M}=\lim _{\frac{G}{l} \rightarrow 0} \frac{G}{l}\left(\frac{c+\bar{c}}{12}\right) .
$$

Now let us focus on the boundary side and look for the equivalent procedure for the flat-space limit in the dual boundary $\mathrm{CFT}_{2}$ of the asymptotically $\mathrm{AdS}_{3}$ spacetimes.

In order to answer this question let us have a closer look at the generic solution (2.2) and try to find the conformal boundary for an arbitrary large $\ell$. The metric of the conformal

\footnotetext{
${ }^{1}$ We should emphasize that the flat-space limit in [8] is different from the modified Penrose limit defined in [16] and the Grassmannian method introduced in [17].
} 
boundary is the same for all $\mathcal{M}$ and $\mathcal{N}$ and is given by:

$$
d s^{2}=\frac{r^{2}}{G^{2}}\left(-\frac{G^{2}}{\ell^{2}} d u^{2}+G^{2} d \phi^{2}\right) .
$$

Thus, $\ell$ can be absorbed in the definition of new time $t=\frac{G}{\ell} u$. The dual CFT lives on a cylinder with coordinates $\{t, \phi\}$ and radius $G$. It is clear that taking the flat-space limit ,$\frac{G}{\ell} \rightarrow 0$, is equivalent to contract time as $t \rightarrow \epsilon t$ with $\epsilon \rightarrow 0$, thus one may guess that the dual of asymptotically flat spacetimes is a CCFT. In two dimensions the symmetry of CCFT is isomorphic to the Galilean conformal algebra(GCA) [7]. In [1, 2], it has been argued that one can obtain the full GCA in two dimensions by contracting the symmetries of the two-dimensional CFT. In this approach the generators of the GCA and the parent CFT and also their central charges are related by

$$
\begin{aligned}
M_{n} & =\lim _{\epsilon \rightarrow 0} \epsilon\left(\mathcal{L}_{n}+\overline{\mathcal{L}}_{-n}\right), \quad L_{n}=\lim _{\epsilon \rightarrow 0}\left(\mathcal{L}_{n}-\overline{\mathcal{L}}_{-n}\right), \\
C_{L L} & =\lim _{\epsilon \rightarrow 0} \frac{c-\bar{c}}{12}, \quad C_{L M}=\lim _{\epsilon \rightarrow 0} \epsilon\left(\frac{c+\bar{c}}{12}\right) .
\end{aligned}
$$

Therefore, the connection between the flat-space limit in the bulk side and contraction of CFT in the boundary side is correct at the level of symmetries and one may propose a dual field theory for the asymptotically flat spacetimes which is a CCFT. We call this duality Flat/CCFT23.

The Falt/CCFT correspondence can be used for finding the quasi local stress tensor of asymptotically flat spacetimes. We expect the same dictionary as the AdS/CFT correspondence i.e. one-point function of energy-momentum operator of the dual CCFT is the stress tensor of the bulk theory. This connection has been addressed in [8] where we have introduced the stress tensor of the asymptotically flat spacetimes using the Flat/CCFT correspondence. The key-point which has been used in [8] is that, therein, the authors used the previously known results about the one-point functions of the GCA and then constructed the flat-space stress tenson. The energy-momentum of field theories which arise by con-

\footnotetext{
${ }^{2}$ Originally this correspondence was coined as BMS/GCA [1, 2] but since for bulk dimensions greater than three the BMS algebra is not exactly GCA while it is still related to a contracted conformal algebra, we call it Flat/CCFT correspondence.

${ }^{3}$ The coordinate which must be contracted can be determined by arguments related to those used for the conformal boundary which has been done in this section. It was time coordinate which needed to be contracted for the dual of AdS written in the BMS gauge. As discussed in [18], for Rindler-AdS one should contract $\mathrm{x}$-coordinate in order to find dual of Rindler spacetime.

${ }^{4}$ The holographic renormalization for the asymptotically flat spacetimes has been also worked out in papers [19] and 20] but non of them explored the connection between flat-space holography and CCFTs.
} 
traction of conformal field theories is given by using energy-momentum tensor of original CFT. For example, for field theories with Galilean conformal symmetry, this connection has been worked out in papers [7]. The CCFT which is dual description of asymptotically flat spacetimes written in the BMS gauge, is defined by contracting time in the original CFT. Thus One would expect that the CCFT Hamiltonian is related to the Hamiltonian of the parent CFT by contracting time, while the momentum operator of both theories are the same, since the $x$-coordinate does not affect by the limiting procedure.

The flat-space stress tensor has been worked out in [8] $]^{5}$.

$$
\tilde{T}_{u u}=\frac{M}{16 \pi G^{2}}, \quad \tilde{T}_{u \phi}=\frac{N}{8 \pi G^{2}}, \quad \tilde{T}_{\phi \phi}=\frac{M}{16 \pi},
$$

where the functions $M$ and $N$ are given by (2.5).

In [8] the above stress tensor (2.13) is used to find conserved charges of symmetry generators $\xi^{\mu}$ by using the Brown and York definition [25]

$$
Q_{\xi}=\int_{\Sigma} d \phi \sqrt{\sigma} v^{\mu} \xi^{\nu} \tilde{T}_{\mu \nu}
$$

where $v^{\mu}$ is the unit timelike vector normal to $\Sigma$. The variation of charges under the symmetry generators yields the $\mathrm{BMS}_{3}$ algebra with exactly the same central extension as [4]. Using the results of [8], we can find the corresponding charges of the generators $M_{0}$ and $L_{0}$ as

$$
Q_{M_{0}}=-\frac{1}{8 G}, \quad Q_{L_{0}}=0
$$

We will use these charges in the next section to compute the entropy of the cosmological solution using the CCFT Cardy-like formula.

\footnotetext{
${ }^{5}$ The results of [8] predict a symmetric structure for the CCFT energy-momentum (EM) tensor. On the other hand one may expect a non-symmetric EM tensor due to absence of Lorentz symmetry for the CCFT. We should emphasize that the symmetric structure of CCFT energy-momentum tensor in the current case is a direct consequence of zero off-diagonal terms for the components of the parent CFT energy-momentum tensor written in the light-cone gauge. Any improvement to the generic case requires a clear understanding of the EM tensor of a theory with Galilean conformal symmetry which may be achieved along the lines of recent papers [21]-24].
} 


\section{A Cardy-Like Formula for the CCFT by Contraction of the Modified Cardy Formula}

Interpreting black holes entropy as degeneracy of microstates of dual theory is an important issue which must be addressed carefully in any gauge/gravity correspondence. The Flat/CCFT correspondence as a duality between gravity in the asymptotically flat spacetimes and Contracted CFTs must provide a clear understanding for the entropy of the asymptotically flat black holes using microstates of the dual CCFT.

Let us concentrate on the Flat $3 / \mathrm{CCFT}_{2}$ and consider the three-dimensional asymptotically flat spacetimes. It was shown in [27] that no asymptotically flat black hole exists in the three-dimensional Einstein Gravity. However, there are other interesting solutions in three dimensional Einstein gravity which have cosmological horizon. They are called cosmological solutions and are given by the following metric:

$$
d s^{2}=\hat{r}_{+}^{2} d t^{2}-\frac{r^{2} d r^{2}}{\hat{r}_{+}^{2}\left(r^{2}-r_{0}^{2}\right)}+r^{2} d \phi^{2}-2 \hat{r}_{+} r_{0} d t d \phi
$$

These solutions are characterized by two parameters $\hat{r}_{+}$and $r_{0}$ which are related to the mass $M$ and the angular momentum $J$ as $\hat{r}_{+}=\sqrt{8 G M}$ and $r_{0}=\sqrt{\frac{2 G}{M}}|J|$. Moreover, $r=r_{0}$ is the radius of the cosmological horizon and one can define the entropy of the cosmological solution by using the area of the cosmological horizon as

$$
S=\frac{A}{4 G}=\frac{\pi r_{0}}{2 G} .
$$

It was shown that the solution (3.1) is the boost-shift orbifold of the three-dimensional Minkowski spacetimes and it can be obtained by taking the flat-space limit of the BTZ black hole [6]. The connection between BTZ black holes and cosmological solutions is important for us in this paper. The idea that the flat-space limit in the bulk corresponds to the contraction of CFT in the boundary can be used to find a Cardy-like formula for the states of CCFT which yields the entropy of the cosmological solution. In fact this idea was first used in paper [5] where a Cardy-like formula was introduced for the $\mathrm{CCFT}_{2}$. The idea of contraction of time for the CFT has been entered in an unusual transformation of the modular parameters of the CCFT. Invariance of the partition function under these new modular transformations along with a saddle point approximation resulted in a formula for the degeneracy of states which correctly reproduces entropy of the cosmological horizon[5]. In this paper we want to argue that the Cardy-like formula of $\mathrm{CCFT}_{2}$ can be obtained by taking a direct contraction 
of the modified Cardy formula of CFT. Before making this connection we need to have a closer look at the bulk and study the flat-space limit of the BTZ black hole.

The cosmological solution is given by taking the flat-space limit of the BTZ black hole with metric

$$
d s^{2}=-\frac{\left(r^{2}-r_{+}^{2}\right)\left(r^{2}-r_{-}^{2}\right)}{r^{2} \ell^{2}} d t^{2}+\frac{r^{2} \ell^{2}}{\left(r^{2}-r_{+}^{2}\right)\left(r^{2}-r_{-}^{2}\right)} d r^{2}+r^{2}\left(d \phi+\frac{r_{+} r_{-}}{\ell r^{2}} d t\right)^{2}
$$

where

$$
r_{ \pm}=\sqrt{2 G \ell(\ell M+J)} \pm \sqrt{2 G \ell(\ell M-J)}
$$

are the radii of the horizons and $M$ and $J$ are related to the mass and the angular momentum of the black hole. It is easy to see that $r_{0}$ in the cosmological solution is given by taking $\ell \rightarrow \infty$ limit of $r_{-}$but $r_{+}$goes to infinity in the flat-space limit. On the other hand according to $\mathrm{AdS}_{3} / \mathrm{CFT}_{2}$ correspondence, the Cardy formula of CFT,

$$
S=2 \pi \sqrt{\frac{c}{6} \mathcal{L}_{0}}+2 \pi \sqrt{\frac{\bar{c}}{6} \overline{\mathcal{L}}_{0}}
$$

results in the correct entropy of the outer horizon $r_{+}$. We can rewrite (3.5) using (2.11) and take $\epsilon \rightarrow 0$ limit which corresponds to $\ell \rightarrow \infty$ limit in the bulk. The final answer diverges as expected because in the bulk side the entropy of the outer horizon diverges in the $\ell \rightarrow \infty$ limit. If we want to find a Cardy-like formula which gives the entropy of the cosmological horizon we should contract a formula in the CFT which corresponds to the entropy of the inner horizon of the BTZ.

The entropy of the inner horizon of the BTZ black hole in terms of the dual CFT parameters, can be written as [12, 13]:

$$
S=\left|2 \pi \sqrt{\frac{c}{6} \mathcal{L}_{0}}-2 \pi \sqrt{\frac{\bar{c}}{6} \overline{\mathcal{L}}_{0}}\right|
$$

Now if we use (2.11) and take the $\epsilon \rightarrow 0$ limit the final answer is well-defined and is exactly the Cardy-like formula which was introduced in [5] i.e.

$$
S=2 \pi\left(C_{L L} \sqrt{\frac{M_{0}}{2 C_{L M}}}+L_{0} \sqrt{\frac{C_{L M}}{2 M_{0}}}\right)
$$

This is another check that flat limit in the bulk corresponds to contraction of CFT in the boundary. 
The Cardy-like formula for the CCFTs can also be given in another form. The point is that there is another form for the Cardy formula (see appendix of [28] and the references therein). Now one can use this new formulation and take limit from it.

The alternative form of the Cardy formula which gives the degeneracy of states of the CFT is given by

$$
S_{C F T}=2 \pi \sqrt{-\left(H_{v a c}+J_{v a c}\right)(H+J)}+2 \pi \sqrt{-\left(H_{v a c}-J_{v a c}\right)(H-J)} .
$$

States are characterized by the eigenvalues of the Hamiltonian and the momentum $(H, J)$ and "vac" denotes the vacuum state. Using the AdS/CFT correspondence, the above formula (3.8) gives the entropy of the outer horizon of the BTZ black holes [28]. However, if we want to find a similar formula for the CCFT, similar to the previous subsection, we should look for a formula in terms of CFT parameters which gives the correct entropy of inner horizon of BTZ black holes. Using [28], one can easily check that the formula

$$
S=2 \pi \sqrt{-\left(H_{v a c}+J_{v a c}\right)(H+J)}-2 \pi \sqrt{-\left(H_{v a c}-J_{v a c}\right)(H-J)}
$$

results in an entropy which matches with the entropy of inner horizon.

Now we can contract (3.9). As discussed in section 2, the Hamiltonian of CCFT is given by contracting the corresponding Hamiltonian of CFT but momenta are the same for both of the theories. If we use this correspondence between $(H, J)$ of $\mathrm{CFT}$ and $(\tilde{H}, \tilde{J})$ of CCFT, we can write

$$
\begin{aligned}
S_{C C F T} & =\lim _{\epsilon \rightarrow 0}\left[2 \pi \sqrt{-\left(\frac{\tilde{H}_{v a c}}{\epsilon}+\tilde{J}_{v a c}\right)\left(\frac{\tilde{H}}{\epsilon}+\tilde{J}\right)}-2 \pi \sqrt{-\left(\frac{\tilde{H}_{v a c}}{\epsilon}-\tilde{J}_{v a c}\right)\left(\frac{\tilde{H}}{\epsilon}-\tilde{J}\right)}\right] \\
& =\lim _{\epsilon \rightarrow 0}\left[2 \pi \frac{-\frac{2}{\epsilon}\left(\tilde{H}_{v a c} \tilde{J}+\tilde{J}_{v a c} \tilde{H}\right)}{\frac{2}{\epsilon} \sqrt{-\tilde{H}_{v a c} \tilde{H}}}\right]=2 \pi \sqrt{-\frac{\tilde{H}_{v a c}}{\tilde{H}}} \tilde{J}+2 \pi \sqrt{-\frac{\tilde{H}}{\tilde{H}_{v a c}}} \tilde{J}_{v a c}
\end{aligned}
$$

Using (2.15), in the bulk side we have

$$
Q_{M_{0}}=\tilde{H}_{v a c}=-\frac{1}{8 G}, \quad Q_{L_{0}}=\tilde{J}_{v a c}=0
$$

where $\tilde{H}_{v a c}$ exactly matches with the free-energy of the cosmological solution that can be obtained from the Euclidean on-shell action [29]. Therefore, from (3.10) the entropy of 
cosmological solution is found to be

$$
S=\frac{\pi}{2 G} J \sqrt{\frac{2 G}{M}}
$$

Using $r_{0}=J \sqrt{\frac{2 G}{M}}$, we end up with the known result of the entropy for the cosmological solution[5] ,

$$
S=\frac{\pi r_{0}}{2 G}
$$

\section{Conclusion}

This work is another check for the correspondence between the flat-space limit in the bulk and contraction of the boundary field theory. We considered the three-dimensional Einstein gravity which admits asymptotically flat geometries, namely cosmological solutions. The asymptotic symmetry group of the asymptotically flat solutions at null infinity is infinite dimensional. The next step is studying the four-dimensional asymptotically flat spacetimes which also have infinite dimensional asymptotic symmetry group[4]. The lessons from the three-dimensional analysis can be used for finding the quasi local stress tensor of the four-dimensional spacetimes and one can also calculate the $\mathrm{BMS}_{4}$ charge algebra using our approach and get insight about the possible central extension of the $\mathrm{BMS}_{4}$ algebra.

Moreover, a Cardy-like formula in the form (3.10) may exist for the $\mathrm{CCFT}_{3}$ which also has infinite dimensional symmetry. We should note that the formulas (3.6) and (3.9) which gives the inner horizon entropy are phenomenological observation and there is not a proof for them in the CFT side. We just wrote the inner horizon entropy of the BTZ in terms of the CFT parameters but what this counting means, is an open question.

\section{Acknowledgement}

The authors would like to especially thank Mahmoud Safari and Seyed Morteza Hosseini for their comments on the manuscript and Stephane Detournay for useful comments. We also thank Arjun Bagchi, Daniel Grumiller and Joan Simon for a continuing collaboration in the flat-spacetime Holography project.

Note added: When this paper was ready for submission, we became aware that Max 
Riegler also has pointed out the limit of the modified Cardy formula which results in (3.7) [30].

\section{References}

[1] A. Bagchi, "Correspondence between Asymptotically Flat Spacetimes and Nonrelativistic Conformal Field Theories," Phys. Rev. Lett. 105, 171601 (2010).

A. Bagchi, "The BMS/GCA correspondence," arXiv:1006.3354 [hep-th].

[2] A. Bagchi and R. Fareghbal, "BMS/GCA Redux: Towards Flatspace Holography from Non-Relativistic Symmetries," JHEP 1210, 092 (2012) [arXiv:1203.5795 [hep-th]].

[3] H. Bondi, M. G. van der Burg, and A. W. Metzner, "Gravitational waves in general relativity. 7. Waves from axisymmetric isolated systems," Proc. Roy. Soc. Lond. A 269 (1962) 21.

R. K. Sachs, "Gravitational waves in general relativity. 8. Waves in asymptotically flat space-times," Proc. Roy. Soc. Lond. A 270 (1962) 103.

R. K. Sachs, "Asymptotic symmetries in gravitational theory," Phys. Rev. 128 (1962) 2851.

[4] G. Barnich and G. Compere, "Classical central extension for asymptotic symmetries at null infinity in three spacetime dimensions," Class. Quant. Grav. 24, F15 (2007) [Erratumibid. 24, 3139 (2007)] arXiv:gr-qc/0610130.

G. Barnich and C. Troessaert, "Symmetries of asymptotically flat 4 dimensional spacetimes at null infinity revisited," Phys. Rev. Lett. 105, 111103 (2010) arXiv:0909.2617 [gr-qc]].

G. Barnich and C. Troessaert, "Aspects of the BMS/CFT correspondence," JHEP 1005, 062 (2010) [arXiv:1001.1541 [hep-th]].

[5] A. Bagchi, S. Detournay, R. Fareghbal and J. Simon, "Holography of 3d Flat Cosmological Horizons," Phys. Rev. Lett. 110, 141302 (2013) arXiv:1208.4372 [hep-th]].

[6] L. Cornalba and M. S. Costa, "A New cosmological scenario in string theory," Phys. Rev. D 66, 066001 (2002) hep-th/0203031.

[7] A. Bagchi and R. Gopakumar, "Galilean Conformal Algebras and AdS/CFT," JHEP 0907, 037 (2009) arXiv:0902.1385 [hep-th]]

M. Alishahiha, A. Davody and A. Vahedi, "On AdS/CFT of Galilean Conformal Field Theories," JHEP 0908, 022 (2009) [arXiv:0903.3953 [hep-th]]. 
A. Bagchi and I. Mandal, "On Representations and Correlation Functions of Galilean Conformal Algebras," Phys. Lett. B 675, 393 (2009) [arXiv:0903.4524 [hep-th]]

A. Bagchi, R. Gopakumar, I. Mandal and A. Miwa, "GCA in 2d," JHEP 1008, 004 (2010) arXiv:0912.1090 [hep-th]].

A. Bagchi, "Topologically Massive Gravity and Galilean Conformal Algebra: A Study of Correlation Functions," JHEP 1102, 091 (2011) [arXiv:1012.3316 [hep-th]].

[8] R. Fareghbal and A. Naseh, "Flat-Space Energy-Momentum Tensor from BMS/GCA Correspondence," JHEP 1403, 005 (2014) [arXiv:1312.2109 [hep-th]].

[9] H. Afshar, A. Bagchi, R. Fareghbal, D. Grumiller and J. Rosseel, "Higher spin theory in 3-dimensional flat space," Phys. Rev. Lett. 111 (2013) 121603 [arXiv:1307.4768 [hep-th]].

[10] H. A. Gonzalez, J. Matulich, M. Pino and R. Troncoso, "Asymptotically flat spacetimes in three-dimensional higher spin gravity," JHEP 1309, 016 (2013) arXiv:1307.5651 [hepth]].

[11] A. Bagchi, R. Basu and A. Mehra, "Galilean Conformal Electrodynamics," JHEP 1411, 061 (2014) [arXiv:1408.0810 [hep-th]].

[12] S. Detournay, "Inner Mechanics of 3d Black Holes," Phys. Rev. Lett. 109, 031101 (2012) arXiv:1204.6088 [hep-th]].

[13] A. Castro and M. J. Rodriguez, "Universal properties and the first law of black hole inner mechanics," Phys. Rev. D 86, 024008 (2012) [arXiv:1204.1284 [hep-th]].

[14] J. Isenberg and V. Moncrief, "Symmetries of Cosmological Cauchy horizons," Commun.Math.Phys. 89 (1983) 387413.

[15] H. Friedrich, I. Racz and R. M. Wald, "On the rigidity theorem for space-times with a stationary event horizon or a compact Cauchy horizon," Commun. Math. Phys. 204, 691 (1999) gr-qc/9811021.

[16] G. Barnich, A. Gomberoff and H. A. Gonzalez, "The Flat limit of three dimensional asymptotically anti-de Sitter spacetimes," Phys. Rev. D 86, 024020 (2012) arXiv:1204.3288 [gr-qc]].

[17] C. Krishnan, A. Raju and S. Roy, "A Grassmann path from $A d S_{3}$ to flat space," JHEP 1403, 036 (2014) [arXiv:1312.2941 [hep-th]]. 
[18] R. Fareghbal and A. Naseh, "Rindler/Contracted-CFT Correspondence," JHEP 1406, 134 (2014) [arXiv:1404.3937 [hep-th]].

[19] R. N. Caldeira Costa, "Aspects of the zero $\Lambda$ limit in the AdS/CFT correspondence," Phys. Rev. D 90, no. 10, 104018 (2014) [arXiv:1311.7339 [hep-th]].

[20] S. Detournay, D. Grumiller, F. Schller and J. Simn, "Variational principle and one-point functions in three-dimensional flat space Einstein gravity," Phys. Rev. D 89, no. 8, 084061 (2014) arXiv:1402.3687 [hep-th]].

[21] M. H. Christensen, J. Hartong, N. A. Obers and B. Rollier, "Boundary Stress-Energy Tensor and Newton-Cartan Geometry in Lifshitz Holography," JHEP 1401, 057 (2014) arXiv:1311.6471 [hep-th]].

[22] K. Jensen, "On the coupling of Galilean-invariant field theories to curved spacetime," arXiv:1408.6855 [hep-th].

[23] J. Hartong, E. Kiritsis and N. A. Obers, "Lifshitz Space-Times for Schroedinger Holography," arXiv:1409.1519 [hep-th].

[24] J. Hartong, E. Kiritsis and N. A. Obers, "Schroedinger Invariance from Lifshitz Isometries in Holography and Field Theory," arXiv:1409.1522 [hep-th].

[25] J. D. Brown and J. W. York, Jr., "Quasilocal energy and conserved charges derived from the gravitational action," Phys. Rev. D 47, 1407 (1993) gr-qc/9209012].

[26] G. Barnich, "Entropy of three-dimensional asymptotically flat cosmological solutions," JHEP 1210, 095 (2012) [arXiv:1208.4371 [hep-th]].

[27] D. Ida, "No black hole theorem in three-dimensional gravity," Phys. Rev. Lett. 85, 3758 (2000) gr-qc/0005129.

[28] S. Detournay, T. Hartman and D. M. Hofman, "Warped Conformal Field Theory," Phys. Rev. D 86, 124018 (2012) [arXiv:1210.0539 [hep-th]].

[29] A. Bagchi, S. Detournay, D. Grumiller and J. Simon, "Cosmic Evolution from Phase Transition of Three-Dimensional Flat Space," Phys. Rev. Lett. 111, no. 18, 181301 (2013) arXiv:1305.2919 [hep-th]].

[30] M. Riegler, "Flat space limit of higher-spin Cardy formula," Phys. Rev. D 91, no. 2, 024044 (2015) arXiv:1408.6931 [hep-th]]. 